\title{
Anatomic thoracoscopic pulmonary segmentectomy under 3-dimensional multidetector computed tomography simulation: A report of 52 consecutive cases
}

Hiroyuki Oizumi, MD, PhD, Naoki Kanauchi, MD, Hirohisa Kato, MD, PhD, Makoto Endoh, MD, PhD, Jun Suzuki, MD, Ken Fukaya, MD, and Mitsuaki Sadahiro, MD, PhD

Objective: The purpose of this retrospective study was to evaluate the efficacy of anatomic thoracoscopic pulmonary segmentectomy performed under the guidance of 3-dimensional multidetector computed tomography simulation.

Methods: Between September 2004 and June 2009, 52 patients (median age, 68 years; range, 16-85 years) underwent thoracoscopic segmentectomy without mini-thoracotomy. Images were obtained by using 64-channel multidetector computed tomography and a contrast agent. The pulmonary arteriovenous structure was mainly determined using a 3-dimensional volume-rendering method. The preoperative simulation was performed at the initial stage of the study and the intraoperative at a later stage. The simulated images were used to identify the venous branches in the affected segment for division and the intersegmental veins to be preserved. Four 5- to 20 -mm ports were used. Segmentectomy was performed by separating the pulmonary arteries and bronchi followed by dissection along the intersegmental plane.

Results: Fifty-one patients underwent a complete thoracoscopic segmentectomy. A mini-thoracotomy was performed in 1 case because of arterial bleeding. The success rate of segmentectomies under complete thoracoscopy was $98 \%$. The procedure was classified into 3 categories according to the degree of surgical difficulty. Before introducing the simulation, there were 4 easy cases and 1 fairly difficult case. After introducing preoperative simulation, 7 cases were classified as fairly difficult among 12 segmentectomy cases. Furthermore, 7 cases of difficult segmentectomy were performed using intraoperative simulation. No local recurrence or metastasis and no mortality were observed during the follow-up.

Conclusions: Thoracoscopic pulmonary segmentectomy under 3-dimensional multidetector computed tomography simulation is a safe technique. (J Thorac Cardiovasc Surg 2011;141:678-82)

Along with the popularization of high-definition devices in recent years, endoscopic surgery has entered into a new period of development. Its demand and indications are increasing in fields other than thoracic surgery. Furthermore, computed tomography (CT) has enabled us to detect small-sized lung cancers. As a result, the numbers of reports of nonsolid tumors appearing as ground glass opacity (GGO), suggesting noninvasive lung cancer with good prognosis, are increasing. ${ }^{1-4}$ Therefore, the procedure for sublobar pulmonary resection has been re-evaluated. Although it is reasonable to perform less-invasive resection of a smaller volume of lung tissue thoracoscopically, com-

From the Second Department of Surgery, Faculty of Medicine, Yamagata University, Yamagata, Japan.

Disclosures: Authors have nothing to disclose with regard to commercial support. Received for publication March 16, 2010; revisions received June 30, 2010; accepted for publication Aug 1, 2010; available ahead of print Sept 30, 2010.

Address reprints to: Hiroyuki Oizumi, MD, PhD, Second Department of Surgery, Yamagata University, 2-2-2 Iida-Nishi, Yamagata 990-9585, Japan (E-mail: hohizumi@ med.id.yamagata-u.ac.jp)

0022-5223/\$36.00

Copyright (c) 2011 by The American Association for Thoracic Surgery doi:10.1016/j.jtcvs.2010.08.027 plex procedures are often involved in this type of surgery. Therefore, reports on segmentectomy under total thoracoscopic vision are limited. ${ }^{5-7}$

We have used three-dimensional (3-D) multidetector CT (MDCT) images for surgical simulation during anatomic thoracoscopic pulmonary segmentectomy. ${ }^{7}$ The aim of this study was to retrospectively evaluate the efficacy of anatomic thoracoscopic pulmonary segmentectomy performed under the guidance of 3-D MDCT simulation.

\section{PATIENTS AND METHODS}

Our institutional ethics committee approved this retrospective study, and the chairperson waived the need for obtaining the consent of individual patients. This study involved 52 consecutive patients $(25$ men and 27 women) who underwent complete thoracoscopic segmentectomy, with written informed consent, between September 2004 and June 2009. Their median age (range) was 68 years (16-85 years).

\section{Selection Criteria}

Our basic selection criteria for thoracoscopic segmentectomy were as follows: (1) patients with intentional resection a T1N0M0 peripheral tumor, GGO of $\leq 2.5 \mathrm{~cm}$ in diameter, and solid tumors with a diameter of $\leq 1.5 \mathrm{~cm}$ with no lymph node metastasis as determined by highresolution $\mathrm{CT}$ in the case of primary lung cancer; these patients had good 


\section{Abbreviations and Acronyms \\ 3-D = three-dimensional \\ CT = computed tomography \\ DICOM = digital imaging and \\ communications in medicine \\ MDCT $=$ multidetector $\mathrm{CT}$ \\ SAMURAI = segmentectomy achieved by \\ MDCT for use in respective anatomical interpretation}

pulmonary function and could tolerate a lobectomy in the management of their disease ${ }^{8}$; (2) patients with compromised resection compromised patients who were considered to be poor candidates for lobectomy because of limited cardiopulmonary reserve or other comorbidities; (3) patients with metastases; and (4) patients with benign lesions.

Wedge resection was considered to be inappropriate in all cases because of the tumor size or location in the deep parenchyma.

\section{Pathology}

There were 37 cases clinically diagnosed as lung cancer but were histologically undiagnosed nodules, 8 cases of metastasis, 5 cases of histologically definitive lung cancer, and 2 cases of benign disease. The patients' characteristics are summarized in Table 1 . In the intentional resection group, the tumor sizes measured with $\mathrm{CT}$ in patients with lung cancer of GGOs were $2.5 \mathrm{~cm}$ in 2 cases and $2 \mathrm{~cm}$ or smaller in 24 cases. The size of all the solid tumors with curative intent for lung cancer was $1.5 \mathrm{~cm}$ or smaller. The largest tumor in the compromised resection group was 2.5 $\mathrm{cm}$ in size. The largest metastatic tumor was $1.7 \mathrm{~cm}$.

\section{Simulated Navigation Method}

We recorded MDCT images by injecting an iodinated contrast medium, and saved the digital imaging and communications in medicine (DICOM) data on a server. We mainly used workstations or a client viewer for image analyses and determined pulmonary arteriovenous structure mainly by using a 3-D volume-rendering method. The surgeon processed the 3-D images within 5 to10 minutes. The window level and window width were adjusted to identify differences in the density of the contrast agent to distinguish between the arteries and the veins. In the absence of such differences, the arteries and veins were distinguished on the basis of measurements, such as the direction of the axis and vessel diameter. The simulation images were used to identify: (1) the segmental arterial branches, (2) the intersegmental veins that were to be preserved, and (3) the venous branches in the affected segment that were to be divided; these data were used to perform the surgical procedure.

In the early stage of this study, we only simulated the procedures on the basis of the images of the vascular structures obtained before the surgery. After September 2007, we saved the DICOM data in personal computers on a Macintosh platform with the Mac OS X 10.4.9 operating system (upgraded later) with a MacBook Pro $2.4 \mathrm{GHz}$ Intel Core 2 Duo (Apple, Cupertino, Calif) and used them in the operating room to create and manipulate similar images during surgery with the free software OsiriX (version 2.7.5, upgraded later; http://www.osirix-viewer.com/Downloads.html). ${ }^{9}$ Thus, we performed the operations while comparing and contrasting the simulation with the real-time conditions in the surgical field by rotating and resizing the 3-D images (Figure 1).

\section{Operative Procedure}

Under the guidance of the previously mentioned simulated navigation technique, we applied Segmentectomy Achieved by MDCT for Use in
TABLE 1. Patient characteristics

\begin{tabular}{lc}
\hline \multicolumn{1}{c}{ Characteristic } & Data (52 cases) \\
\hline Age in years (range) & $66 \pm 13(16-85)$ \\
Male/Female & $25 / 27$ \\
Clinical diagnosis & \\
Undiagnosed nodule & 37 \\
$\quad$ CT shadow & \\
$\quad$ Nonsolid/solid & $28 / 9$ \\
Pathology & \\
$\quad$ Nonsolid & \\
$\quad$ Adenocarcinoma & 26 \\
$\quad$ Capillary hemangioma & 1 \\
$\quad$ Inflammation & 1 \\
$\quad$ Solid & \\
$\quad$ Adenocarcinoma & 3 \\
$\quad$ Squamous cell carcinoma & 4 \\
$\quad$ Small-cell carcinoma & 1 \\
$\quad$ Anthracotic nodule & 1 \\
Lung cancer & 5 \\
Adenocarcinoma & 3 \\
Squamous cell carcinoma & 2 \\
Metastasis & 8 \\
Benign & 2 \\
FVC $(\%)$ & $75 \pm 9$ \\
FEV1 (\%) & \\
\hline
\end{tabular}

Results are expressed as mean \pm standard deviation. $C T$, Computed tomography; $F E V 1$, forced expiratory volume in 1 second; $F V C$, forced vital capacity.

Respective Anatomical Interpretation (SAMURAI). We collapsed the lung on the operating side to anesthetize the patients under differential ventilation. During the procedure, the surgeons stood on the ventral side of the patient and the assistants stood on the dorsal side. The image, on the side of the assistants, was rotated 180 degrees. Four ports ( 1 with a diameter of 20 or 15 $\mathrm{mm}$ [flexible], and 3 with a diameter of $5 \mathrm{~mm}$ ) were prepared for the portaccess technique. We first ligated and dissected the segmental artery and then detached the bronchus. In general, we ligated the bronchus or dissected it with a stapler after inflation of the lung on the operating side. The inflationdeflation lines became gradually clear by recollapsing the lung. We first identified the intersegmental veins while tugging the bronchi for intersegmental dissection. Then we dissected the pulmonary parenchyma along the intersegmental pulmonary veins using an electrocautery or ultrasonically activated device guided by the inflation-deflation lines. The veins were detached from the hilum toward the peripheral side after deciding which intersegmental pulmonary veins were to be preserved and which were to be dissected on the basis of the simulation. The peripheral lungs and pulmonary parenchyma that were sufficiently distal to the tumor were dissected with stapling devices.

In cases of suspected malignancy, we designed the resection lines with a larger surgical margin than the tumor diameter (at least $1 \mathrm{~cm}$ or more even for a tumor smaller than $1 \mathrm{~cm}$ ). If it was difficult to preserve the margin in a single segment resection, we performed an extended segmentectomy by dissecting the parenchyma across the intersegmental vein, or combined segmentectomy with the adjacent subsegments. We also performed block resection of multiple segments if the size of the tumor with GGO was > $2 \mathrm{~cm}$, the size of the solid tumor was $>1 \mathrm{~cm}$, or the tumor had pleural indentations or vascular convergence, even if it was $<1 \mathrm{~cm}$. In addition, we performed lymph node sampling instead of dissection in the case of lung cancer presenting with pure GGO and mediastinal lymph node dissection in the case of solid tumors and tumors with solid regions and GGO. The resected segments were removed in a retrieval bag. In addition, we applied fibrin glue or intracavitary continuous sutures on the surface of the dissected segment as appropriate. 

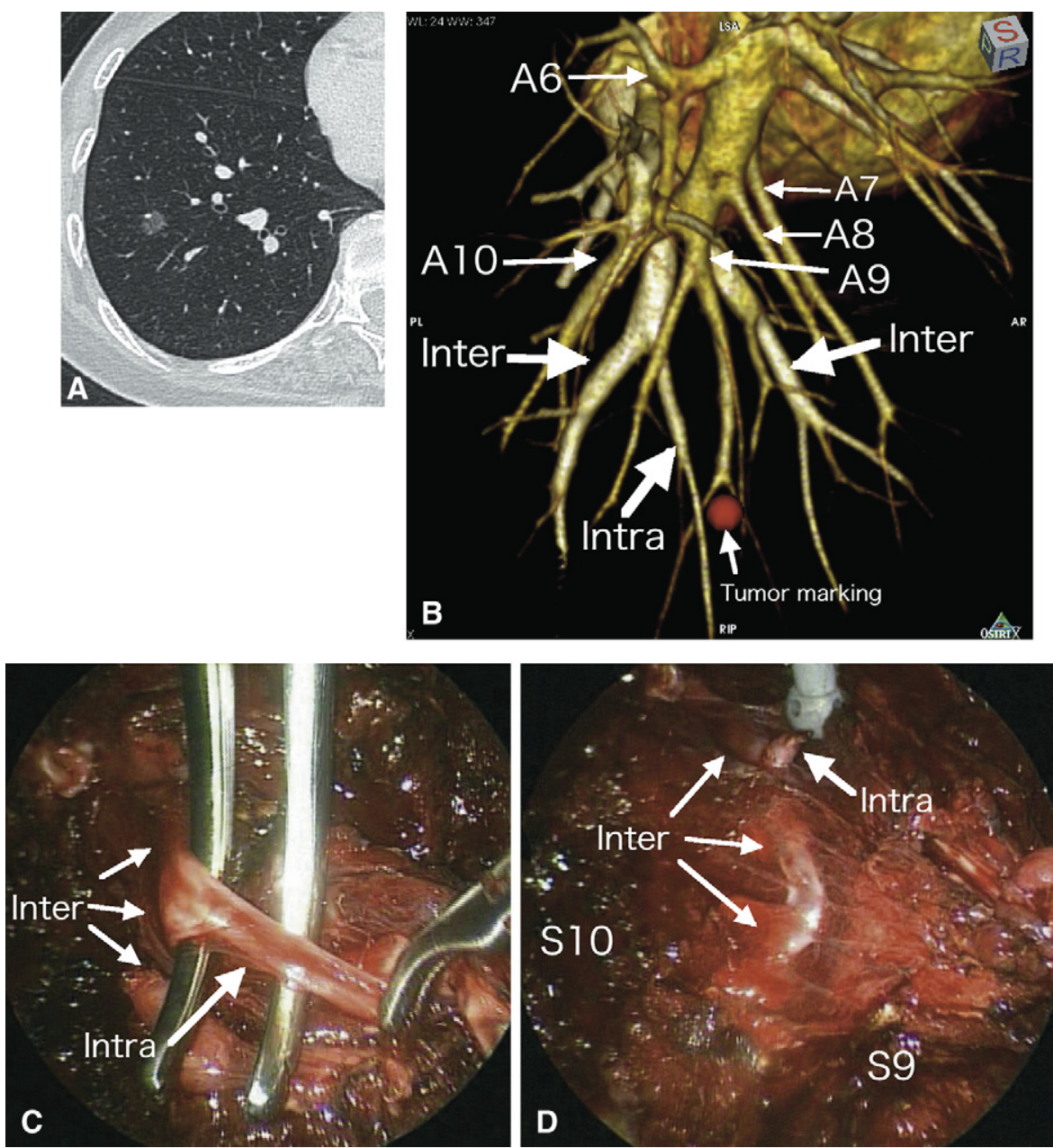

FIGURE 1. The lateral basal segment (S9) resection of the right lower lobe, a representative case of difficult segmentectomy. A, The computed tomographic (CT) image showing a 1.1-cm ground glass opacity (GGO) (adenocarcinoma) located in S9. B, Three-dimensional computed tomography angiography with a marking of the tumor. (A6, Segmental artery of S6; A\#, indicates the corresponding segments; inter, intersegmental vein; intra, intrasegmental vein.) $\mathrm{C}$ and $\mathrm{D}$, Operative view of the patient. The intrasegmental vein was divided and the intersegmental plane was dissected preserving the intersegmental vein.

\section{RESULTS}

\section{Surgical Outcome}

Table 2 summarizes the resected segments according to the nomenclature previously described. ${ }^{10}$ A lobectomy was performed in one case after completing the segmentectomy because small-cell carcinoma was detected by frozen section. The surgical results of the remaining 51 cases were as follows: operating time, median of 216 minutes (range, 115-425 mins); volume of hemorrhage, median of $66 \mathrm{~mL}$ (range, 0-517 $\mathrm{mL}$ ); duration of chest tube insertion, median of 1 day (mean \pm standard deviation $=2.1 \pm 1.7$; range, $1-8$ days); and complications, one case each of the ulceration of the left main bronchus due to heat injury by electrocautery during subcarinal lymphadenectomy, subcutaneous emphysema, and late pneumothorax (postoperative day 8). In one case, we extended the incision to $12 \mathrm{~cm}$ to achieve hemostasis after hemorrhage $(280 \mathrm{~mL})$ from the pulmonary artery.
The success rate of segmentectomy under complete thoracoscopy was $98 \%$.

Of the 38 cases of lung cancer, hilar lymphadenectomy was performed in 16 cases, hilar and mediastinal lymphadenectomy in 12 cases, and lymphadenectomy was not performed in 10 cases. No lymph node metastasis was observed in any of the cases, and the pathologic stage was T1NOM0. No recurrence and no mortality were observed during the follow-up period of as $<5$ years and 3 months (mean, 2 years, 7 months).

\section{Procedural Classification}

We classified thoracoscopic segmentectomy into 3 categories according to the degree of procedural difficulty as follows:

Easy: A single intersegmental dissection surface, or reports of similar cases of thoracoscopic surgery. 
TABLE 2. Resected segments

\begin{tabular}{|c|c|c|c|}
\hline Right & $N$ & Left & $N$ \\
\hline S1 & 3 & $\mathrm{~S} 1+2$ & 4 \\
\hline $\mathrm{S} 2$ & 5 & $\mathrm{~S} 1+2,3$ & 8 \\
\hline $\mathrm{S} 2,3 \mathrm{a}$ & 1 & $\mathrm{~S} 1+2,3,6$ & 1 \\
\hline $\mathrm{S} 3$ & 2 & $\mathrm{~S} 3,4$ & 1 \\
\hline $\mathrm{S} 3,1 \mathrm{~b}$ & 1 & $\mathrm{~S} 4,5$ & 2 \\
\hline S6 & 5 & & \\
\hline S6* & 1 & & \\
\hline S8 & 3 & S8 & 4 \\
\hline S9 & 1 & S9 & 1 \\
\hline S8,9 & 1 & S8,9 & 1 \\
\hline $\mathrm{S} 9,10$ & 3 & $\mathrm{~S} 8,9,10$ & 4 \\
\hline Total & 26 & & 26 \\
\hline
\end{tabular}

Fairly difficult: Multiple dissection surfaces contact at obtuse angles, or there are no similar reported cases of thoracoscopic surgery even with a single dissection surface.

Difficult: Multiple dissection surfaces at acute angles, or a bronchial pulmonary artery located deep within the segment.

Before introducing the simulated navigation technique, most of the cases were classified as easy. After we introduced SAMURAI, the number of segmentectomies classified as fairly difficult increased, accounting for more than half of the cases. Furthermore, we performed 7 segmentectomies, classified as difficult by using intraoperative simulated navigation (Figure 2).

\section{DISCUSSION}

Most of the reports on thoracoscopic pulmonary segmentectomy are usually limited to anatomically easy segments, such as lingular segments. ${ }^{5,6,11,12}$ Intersegmental dissection in such segmentectomies is not procedurally difficult, because the dissection can simply be performed by using stapling devices along the intersegmental plane. However, this procedure is difficult to apply to other segments. For most intersegmental surfaces, a thoracotomy or minithoracotomy is necessary. Both involve dissection by relying on tactile sense or direct vision. Formerly, videoassisted thoracoscopic lobectomies have been performed in some cases of GGO, if requested by patients.

The factors influencing the outcome of thoracoscopic pulmonary segmentectomy are intersegmental determination and difficulty in dissection. Visualization of the inflation-deflation lines is reportedly useful for intersegmental determination and dissection, and the procedure is now applied for less-invasive surgery; however, mini-thoracotomy is required for direct visualization. ${ }^{13}$ In addition, it is not easy to visualize the inflation-deflation lines in emphysematous lungs, such as in the case of chronic pulmonary emphysema. The use of CT is rapidly being replaced by MDCT to facilitate easier diagno-
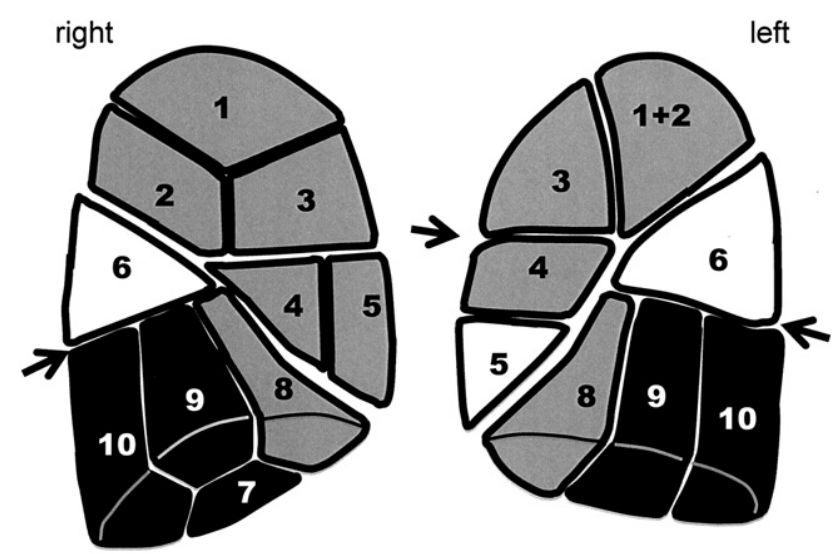

\begin{tabular}{cccc} 
Level & Sim $(-)$ & Preop Sim & Intraop Sim \\
\hline$\square$ Easy & 4 & 4 & 11 \\
$\square$ Fairly & 1 & 7 & 17 \\
difficult & 17 & 7 \\
\hline Difficult & 0 & 1 & 35 \\
\hline Total & 5 & 12 & 7
\end{tabular}

FIGURE 2. A diagram of the segments and numbers of segmentectomies according to the difficulty classification system. (Arrow, Easy plane; Sim [-], before the introduction of simulation; Preop Sim, after the introduction of preoperative simulation; Intraop Sim, after the introduction of intraoperative simulation.)

sis and visualization of the anatomy on the basis of 3-D imaging. ${ }^{14-16}$ SAMURAI is a segmentectomy technique in which the location of the tumor resection margin is estimated on the basis of CT images, and the target blood vessels are determined in $3-\mathrm{D} .{ }^{7}$ Using this procedure to identify the intersegmental veins and then dissecting along them, one can perform segmentectomy via a completely thoracoscopic approach and can be performed even for anatomically difficult segments. ${ }^{5,6,11,12}$ This procedure does not cost more because contrast medium-enhanced CT is performed routinely to evaluate lymph node enlargement in lung cancer patients in our institution.

The maximum operating time in this series was 425 minutes for 1 case that was classified as difficult. In this case, segmentectomy was performed by using only preoperative simulation. As this operating time is unacceptable for pulmonary segmentectomy, we performed this complicated segmentectomy by bringing a laptop computer into the operating room and manipulating the 3-D images with a mouse sterilized with ethylene oxide gas. The use of such real-time images is inexpensive because of the use of free software and reproduction time are as short as 5 minutes. Once the 3-D reconstruction is created, the image can be used repeatedly as required. The operating time was subsequently shortened to $<300$ minutes in difficult cases. There are many biases, such as difficulty, learning curve, and so forth. Lately, inexperienced surgeons performed most of the 
easy segmentectomies in our institution. Therefore it may be inappropriate to simply compare the operating times. According to our data, if limited to easy segmentectomies, operating times of pre- and post-simulation were $209 \pm 50$ and $185 \pm 37$ minutes, respectively. Furthermore, if the operator is limited to the experienced surgeon, operating time was $176 \pm 32$ minutes, which may indicate the merit of simulation. The long operating time can be attributable to the learning curve involved, and it is possible that the operating time would reduce as the procedure becomes standardized in the future.

The number of reports on small-sized lung cancer, which is believed to be noninvasive, has recently increased. It is increasingly being recognized that wedge resection of these tumors results in a good prognosis, especially in the case of nonsolid tumors. ${ }^{1-4}$ In the case of noninvasive lung cancers located deep within the pleura on the hilar side, it is impossible to perform thoracoscopic resection with sufficient preservation of the margin. Segmentectomy can be considered in such cases, as well as in compromised patients. We consider that pulmonary function can be preserved to a greater degree by combining segmentectomy with less-invasive thoracoscopic surgery. Although this is only a preliminary report on thoracoscopic SAMURAI, no recurrence or mortality was observed in any of the cases, including the first case of an 80-year-old female patient with compromised indication, implying the significant potential of this technique.

The hospital picture archiving and communication system has recently been improved, and it is now easier to view 3 -D images in the operating room. With improvements in endoscopic surgery instruments and imaging techniques, the use of this procedure for pulmonary resection is expected to increase.

\section{References}

1. Okada M, Nishio W, Sakamoto T, Uchino K, Hanioka K, Ohbayashi C, et al. Correlation between computed tomographic findings, bronchioloalveolar carcinoma component, and biologic behavior of small-sized lung adenocarcinomas. J Thorac Cardiovasc Surg. 2004;127:857-61.

2. Yoshida J, Nagai K, Yokose T, Nishimura M, Kakinuma R, Ohmatsu H, et al. Limited resection trial for pulmonary ground-glass opacity nodules: fifty-case experience. J Thorac Cardiovasc Surg. 2005;129:991-6.

3. Nakata M, Sawada S, Yamashita M, Saeki H, Kurita A, Takashima S, et al. Objective radiologic analysis of ground-glass opacity aimed at curative limited resection for small peripheral non-small cell lung cancer. $J$ Thorac Cardiovasc Surg. 2005;129:1226-31.

4. Mun M, Kohno T. Efficacy of thoracoscopic resection for multifocal bronchioloalveolar carcinoma showing pure ground-glass opacities of $20 \mathrm{~mm}$ or less in diameter. J Thorac Cardiovasc Surg. 2007;134:877-82.

5. Shiraishi T, Shirakusa T, Iwasaki A, Hiratsuka M, Yamamoto S, Kawahara K. Video-assisted thoracoscopic surgery (VATS) segmentectomy for small peripheral lung cancer tumors: intermediate results. Surg Endosc. 2004;18:1657-62.

6. Atkins BZ, Harpole DH Jr, Mangum JH, Toloza EM, D'Amico TA, Burfeind WR Jr. Pulmonary segmentectomy by thoracotomy or thoracoscopy: reduced hospital length of stay with a minimally-invasive approach. Ann Thorac Surg. 2007;84:1107-12.

7. Oizumi H, Kanauchi N, Kato H, Endoh M, Takeda S, Suzuki J, et al. Total thoracoscopic pulmonary segmentectomy. Eur J Cardiothorac Surg. 2009;36:374-7.

8. Kodama K, Doi O, Higashiyama M, Yokouchi H. Intentional limited resection for selected patients with T1 N0 M0 non-small-cell lung cancer: a singleinstitution study. J Thorac Cardiovasc Surg. 1997;114:347-53.

9. Rosset C, Rosset A, Ratib O. General consumer communication tools for improved image management and communication in medicine. J Digit Imaging. 2005; 18:270-9.

10. Boyden EA. A critique of the international nomenclature on bronchopulmonary segments. Dis Chest. 1953;23:266-9.

11. D'Amico TA. Thoracoscopic segmentectomy: technical considerations and outcomes. Ann Thorac Surg. 2008;85:S716-8.

12. Shapiro M, Weiser TS, Wisnivesky JP, Chin C, Arustamyan M, Swanson SJ. Thoracoscopic segmentectomy compares favorably with thoracoscopic lobectomy for patients with small stage I lung cancer. J Thorac Cardiovasc Surg. 2009;137:1388-93.

13. Okada M, Mimura T, Ikegaki J, Katoh H, Itoh H, Tsubota N. A novel videoassisted anatomic segmentectomy technique: selective segmental inflation via bronchofiberoptic jet followed by cautery cutting. J Thorac Cardiovasc Surg. 2007; 133:753-8.

14. Lamade W, Vetter M, Hassenpflug P, Thorn M, Meinzer HP, Herfarth C. Navigation and image-guided HBP surgery: a review and preview. J Hepatobiliary Pancreat Surg. 2002;9:592-9.

15. Troulis MJ, Everett P, Seldin EB, Kikinis R, Kaban LB. Development of a threedimensional treatment planning system based on computed tomographic data. Int J Oral Maxillofac Surg. 2002;31:349-57.

16. Fukuhara K, Akashi A, Nakane S, Tomita E. Preoperative assessment of the pulmonary artery by three-dimensional computed tomography before video-assisted thoracic surgery lobectomy. Eur J Cardiothorac Surg. 2008;34:875-7. 